Visión Antataura

Vol.4, No.1: 1-24

Junio - Noviembre, 2020

Panamá

ISSN 2520-9892

\title{
Tejiendo la memoria del agua: La re-significación de ecosistemas estratégicos en términos de paisaje cultural
}

\section{Weaving the memory of water: The re-significance of strategic ecosystems in terms of cultural landscape}

\author{
Mauricio Javier Sierra Morales ${ }^{1}$
}

\begin{abstract}
${ }^{1}$ Post-doctorado en Ciencias Sociales, Doctorado en Urbanismo, Maestría en Planificación y Administración del Desarrollo Regional, Profesional en Arquitectura; Docente Titular, Universidad de Boyacá, Tunja, Colombia; majasimo@gmail.com; https://orcid.org/0000-0002-0486-0417
\end{abstract}

Resumen: Somos predominantemente agua: onda y materia simultáneamente interactuando a niveles cuánticos y cósmicos respecto al mundo que habitamos y, es nuestra consciencia la que se mueve, tanto a diversas escalas fractales como en múltiples dimensiones de nuestra existencia. Podemos afectar la mente colectiva planetaria y corregir errores de comportamiento en los seres humanos, desde la intención hasta la acción, modificando la forma del agua y tejiendo la memoria colectiva de humanos y no humanos. El error primordial de la presente humanidad consiste en haber reemplazado la cultura matrística original, basada en el poder del cuidado, por una cultura occidental dominante, jerárquica, separatista basada en el poder patriarcal. Antes de la llegada de dicha manera de pensar y actuar a nuestro continente, hubo otras civilizaciones, mundos enteros olvidados, como la cultura Zenú de la depresión geográfica momposina colombiana, cuya cultura matrística había logrado armonizar el tejido del agua mediante infraestructuras hidráulicas capaces de garantizar la seguridad y soberanía alimentaria, habitar de manera segura y confortable el territorio, aprovechar eficaz y eficientemente los recursos de la naturaleza y de paso respetarla y promover el bienestar a todas las especies no humanas a escala continental.

Palabras clave: Memoria del agua, ecosistemas estratégicos, paisaje cultural, fronteras e interfaces.

Abstract: We are predominantly water, we are the wave and matter simultaneously interacting at quantum and cosmic levels with respect to the world we inhabit in and it is our consciousness that moves, both at various fractal scales and in multiple dimensions of our existence. We can affect the planetary collective mind and correct behavioral errors in humans, from the intention to the action, modifying the water shape and weaving the collective memory of human beings and nonhuman beings. The primordial error of the present humanity consists of having replaced the original matristic culture, based on the care power, with a dominant, hierarchical, separatist western culture based on patriarchal power. Before the arrival of this way of thinking and acting in our continent, there were other forgotten civilizations, and entire worlds, such as the Zenú culture of the Colombian Momposina geographical depression, whose matristic culture had managed to harmonize the tissue of water through hydraulic infrastructures capable of guaranteeing food security and sovereignty, inhabiting the territory in a safe and comfortable way, taking advantage of the resources of nature effectively and efficiently and by all means respecting it and promoting the well-being of all nonhuman species in a continental scale.

Key words: Memory of water, strategic ecosystems, cultural landscape, borders and interfaces. 


\section{Introducción:}

"Tejiendo la Memoria del Agua" es resultado de una investigación que relaciona ecosistemas estratégicos - como los humedales y los páramos - con la idea de paisaje cultural, en términos de la Organización de las Naciones Unidas para la Educación, la Ciencia y la Cultura (UNESCO) y la declaratoria de Sistemas Importantes del Patrimonio [Alimentario] Mundial - (SIPAM)², de la Organización de las Naciones Unidas para la Agricultura y la Alimentación / Organización de las Naciones Unidas (FAO / ONU).

Una de las facultades más sorprendentes del agua es su capacidad de almacenar memoria. El investigador japonés Masaru Emoto (2012), demostró mediante la microfotografía que, a nivel molecular, el agua se organiza en patrones armónicos de gran belleza o fealdad, dependiendo del mensaje escrito que, a su vez, representa pensamientos de amor o armonía y de discordia u odio respectivamente.

Así como el agua es capaz de "recordar" acontecimientos, también es posible para nosotros los humanos decodificarla. Finalmente, somos un setenta por ciento agua, al igual que la superficie terrestre: setenta por ciento es agua.

Las energías sutiles relacionadas con la consciencia, el $\mathrm{HADO}^{3}$ o patrón vibracional cuántico de toda la materia, son capaces de materializarse hasta el punto de satisfacer las necesidades y deseos más humanos. ¿Cómo algo tan simple, desde el punto de vista físicoquímico como el agua puede contribuir al desarrollo en términos más complejos y humanos?

$2 \quad$ Los "Sistemas Importantes del Patrimonio Agrícola Mundial" (SIPAM) son paisajes turísticamente interesantes, biodiversos, cuyos ecosistemas resilientes representan un valioso patrimonio cultural para la humanidad. Situados estratégicamente aportan de manera sostenible diversidad de bienes y servicios que constituyen el soporte bio-físico y los medios de subsistencia para millones de habitantes, así como la base productiva de pequeños agricultores (incluida la pesca y la ganadería), de manera que garantizan la seguridad y soberanía alimentaria de campesinos y citadinos. El término [alimentario] - más amplio - en lugar del término "agrícola" se debe a que la presente investigación trata de ecosistemas estratégicos hídricos y su correspondiente producción acuícola, aunque no excluye la agricultura e incluso la ganadería a pequeña escala.

3 Verónica. (21.07.2011). La consciencia afecta a la materia. Masuro Emoto. Chile: Blog de crecimiento personal. Recuperado de: <http://mujerayuda.blogspot.com/2011/07/la-consciencia-afecta-la-materia$\underline{\text { masaru.html> }}$ 
¿Será posible, imprimir mediante diseños geométricos complejos la memoria del agua almacenándola en forma de energía cósmica? La posibilidad de que las extrañas propiedades cuánticas de la materia (recursividad, hologramía, dialogicidad, entre otras), afecten la realidad macroscópica donde se manifiesta la consciencia, depende de un ajuste o enfoque a nuestra mirada de lo mirado. No se trata de corregir nuestra epistemología sino cambiar nuestra ontología.

Influir en la estructura del agua y, con ello también en la memoria, puede contribuir a corregir errores de comportamiento de las personas a partir de la forma. Esta es la asombrosa hipótesis de trabajo de la presente investigación denominada: Tejiendo la Memoria del Agua.

Lo anterior, es equivalente a desarrollar maneras de corregir Gaia. Suponiendo que sea cierta la hipótesis de James Lovelock (1985), quien concibe el planeta entero, la tierra, como un organismo viviente. Este macroorganismo vivo es capaz de albergar vida debido a su capacidad de autoregulación. ¿Somos nosotros, los humanos, la mente de Gaia?

Si la respuesta a la anterior pregunta es afirmativa, entonces estamos ante un gravísimo error de auto programación a escala planetaria. Es decir, si aceptamos la idea de que no podemos dejar al azar de la vida o a la voluntad divina el futuro, no solo de la humanidad, sino del planeta entero como organismo viviente, entenderemos inmediatamente que no se trata solamente de la mente en términos científicos, sino la mente de quienes habitamos el planeta.

Tendríamos entonces que reconocer - con humildad - que no somos los primeros en este planeta en darnos cuenta de que "el consumo desmesurado de combustibles fósiles, además de modificar la química de la atmósfera y la temperatura "global" del planeta, induce en la Tierra un proceso de "retroalimentación" que hace subir la temperatura casi en espiral, rompiendo este mecanismo de "autorregulación" para la preservación de la vida..." (Lovelock, 2000).

Antes de nosotros, y, es lo que intenta demostrar la presente investigación, hubo otras humanidades que probablemente intentaron corregir también sus propios errores; aunque quizá un poco tarde. Nos interesa descubrir hasta dónde lograron avanzar en dicho 
4. Visión Antataura, Vol.4, No.1, Junio - Noviembre 2020

propósito y si lograron, de alguna manera, corregir errores del comportamiento de los seres humanos modificando intencionalmente la forma del agua y tejiendo, entre todos, humanos y no humanos, la memoria colectiva.

\section{La re-significación de ecosistemas estratégicos en términos de paisaje cultural.}

La memoria del agua es la teoría que sustenta la idea que el hábitat humano contemporáneo carece de sentido; fundamentalmente debido a que existe un vacío de poder, precisamente en términos humanos. Lograr la sustentabilidad del hábitat como fenómeno observable requiere primero que haya un cambio en la persona que lo observa, en su manera de mirar, más allá de las diferencias de género y criterio. Sin embargo, dicho cambio no termina ahí. Se requiere, además, cambiar el sentido humano de la observación; la diferencia entre objeto y sujeto en términos de omnijeto. Un enfoque además libre de las restricciones del espacio y por tanto del tiempo: un pasado-futuro.

Los camellones indígenas del pueblo Zenú no fueron construidos en el pasado, son lo pasado que nos espera al futuro probable y para lograrlo, debemos primero entenderlos como ecosistemas estratégicos y, resignificarlos como paisaje cultural, como patrimonio de la humanidad. El pueblo que los construyó no lo hizo "antes" de nosotros. Lo vamos juntos a construir en el futuro, como sistemas importantes de ese patrimonio mundial y, aunque no suene creíble para nuestro ego tecnológicamente agrandado, lo será para asegurar la subsistencia alimentaria.

Las siguientes premisas, a modo de supuestos verificables, lo son o no (verificables), dependiendo de que mantengamos (o no), la consciencia en sintonía con aquella frecuencia de alternatividad: otro tiempo y, por tanto, otro espacio para la memoria.

Premisa 1: El vacío de maternidad en nuestras estructuras de poder patriarcales es similar al vacío de poder en la especie humanidad. 
En un fragmento del documental "Nacimiento Orgásmico" ${ }^{4}$ la ginecóloga Christiane Northrup (2010), explica cómo el parto en el agua, llevado a cabo de manera natural y asistido por comadronas expertas, tal y como era en épocas cuando la humanidad se dominaba de manera matrística, recupera parte de la sexualidad femenina reprimida, eliminando de paso prejuicios y teorías que deberían estar hoy obsoletas, así como los miedos que la mujer adquirió al dolor y al funcionamiento natural de su propio cuerpo.

La matrística es una forma de organización socio-afectiva que se rige por el principio materno: es un tipo de amor filo-genéticamente diseñado como fuente de energía empática. Los grupos humanos formados en estos núcleos se basan en el cuidado mutuo, en la fraternidad humana, la hospitalidad al extranjero, la paz tan anhelada que hemos perdido. La gran madre era venerada y las mujeres eran sus sacerdotisas. Figuras que datan de la edad de piedra encontradas en la Europa antigua, Asia, y las Américas representan la naturaleza y, su cuerpo la tierra misma: el milagro de la vida.

Figura 1. Cultura Matrística Original.

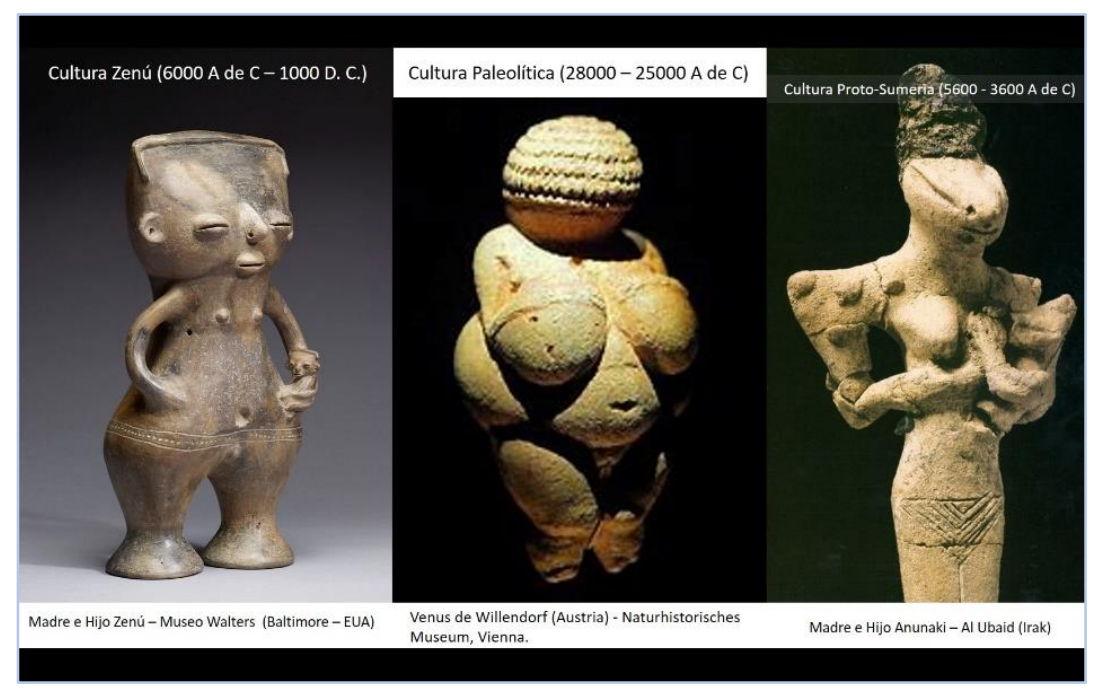

Fuentes: Walters Museum. Baltimore (E.U.A.) / Naturhistorisches Museum, Vienna (Austria) / Excavaciones de Harry Reginald Hall y Sir Charles Leonard Woolley en Tell al'Ubaid.

\footnotetext{
$4 \quad$ Para conocer más acerca de la experiencia del nacimiento por parto natural y en el agua, se recomienda el Blog de Asta. (17.03.2020). Orgasmic Birth. The Best-Kept Secret - The Film. New York: Orgasmic Birthing. Recuperado de: <https://www.orgasmicbirth.com/products/films-soundtrack/>
} 
Lo contrario es una sociedad endurecida en la guerra, basada en el régimen de esclavitud. Una humanidad corrompida y cuyo principio se basa en separar la madre de sus criaturas. Para el logro de lo anterior, hay que señalar que el calostro es dañino y que la mujer es impura por haber parido.

La casta sacerdotal masculina se encargó de tal misión: separar de facto las generaciones y crear el rechazo de la madre a sus propios deseos pos-parto; llegando incluso a afirmar, como en la era victoriana, que "el demonio estaba en la leche materna".

Eliminar el placer de la mujer en el parto e inhibir el orgasmo a partir del útero fue el primer paso para lograr el dominio patriarcal. Las implicaciones en el campo de la neurología son impresionantes. Como sabemos, el humano nace apenas con el 80 por ciento del cerebro formado. Sin ese contacto temprano con la madre no se forma correctamente la sinapsis (interrelación neuronal) en el humano, lo cual explica en grande medida nuestra desconexión con la naturaleza, con Gaia.

Pero, la cosa no acaba ahí: al no haber desarrollado la capacidad neurotransmisora de respuesta al estrés emocional que supone para la cría el estar separado de la madre, se genera un patrón de respuesta neuroquímico, hormonal, favorable a la violencia, a la esclavitud y a la guerra.

La maternidad como desarrollo de la sexualidad femenina ha sido reprimida y con ello, se ha inhibido la capacidad de recibir la hormona oxitocina en el cuerpo ${ }^{5}$. En una entrevista clave, Casilda Rodrigáñez ${ }^{6}$, afirma que la consecuencia es el dolor en el parto: el mal llamado castigo divino, que no es otra cosa que un mal programado por la sociedad patriarcal.

5 La oxitocina tiene la capacidad de producir empatía, representa la oportunidad de reconocer las emociones de los otros y de comportarnos afectivamente; actuando como mediador químico que produce y sostiene el comportamiento maternal en la mujer y paternal en el hombre. Belén Igual. (26.04.2017). ¿Qué es la Oxitocina? : Blog CuerpoMente. Recuperado de: $\leq$ https://www.cuerpomente.com/salud-natural/terapiasnaturales/que-es-hormona-oxitocina-para-que-sirve 264>

$6 \quad$ El poder femenino radica en su sexualidad, en su sentido más amplio. Se recomienda ver el vídeo de la entrevista a Casilda Rodrigañez. Ibone Olza. (23.10.2018). Charla Casilda Rodrigañez. Barcelona: Instituto Europeo de Salud Mental Perinatal. Recuperado de: <https://www.youtube.com/watch?time_continue=14\&v=T9kBRA0RQ38> 
La verdadera emancipación femenina significa recuperar el sentido de maternidad como desarrollo de la sexualidad. Esto último tiene grandes implicaciones en la cultura del agua, que como ya explicamos, es de naturaleza femenina. Ahora bien, entendiendo humanidad, más allá de lo femenino y lo masculino, recuperar el sentido de maternidad implica la paternidad masculina como desarrollo de la sexualidad. Femeneidad y masculinidad no es un asunto - exclusivamente - sexual.

Premisa 2: Los camellones indígenas Zenúes no pertenecen a la historia "oficial", son relatos de un pasado "futuro".

La re-significación es un procedimiento interactivo que parte del supuesto de que existe - una interpretación errada de los hechos, lo cual explica - mas no disculpa - la falta de consciencia. Suponemos, que vamos a darle - a los hechos - un significado nuevo, cuyo valor radica, no en la novedad, sino en la veracidad del significante, más que la verosimilitud del significado.

El uso de la semiótica como estrategia para darle un significado - supuestamente nuevo - al rol de la mujer en la sociedad contemporánea, implica además de recuperar la cultura matrística original, relacionar a "ella" con lo femenino del agua.

La palabra agua es femenina. En idioma español no decimos como en portugués "a agua" quizá por evitar el hiato a-a. Pero el "error" intencionado va más allá de la mera gramática. Toda la naturaleza es femenina. Siguiendo con el ejemplo, en portugués se dice "a árvore" en lugar de decir "el árbol". Todo el lenguaje está cargado de significado e intencionalidad y por esto, no es casual que, para plantear una posible re-significación de los ecosistemas estratégicos, en este caso los humedales transformados en paisaje cultural, quizá debamos re-pensar la idea del tiempo (y por tanto del espacio) como marcos o referentes ontológicos de esa supuesta realidad o verdad.

En la siguiente figura, se observa una maqueta de un posible objeto volador (aún) no identificado - OVANO -, y un Artefacto precolombino Zenú expuesto al público en el Museo del Oro de Bogotá Colombia. ¿Se trata de una coincidencia que los científicos han 
catalogado como la representación de un animal volador, o realmente podría representar algo que los antiguos habitantes del territorio Zenú "vieron" sobrevolando los camellones?

\section{Figura 2. Artefacto precolombino Sinú en el Museo del Oro de Bogotá Colombia}

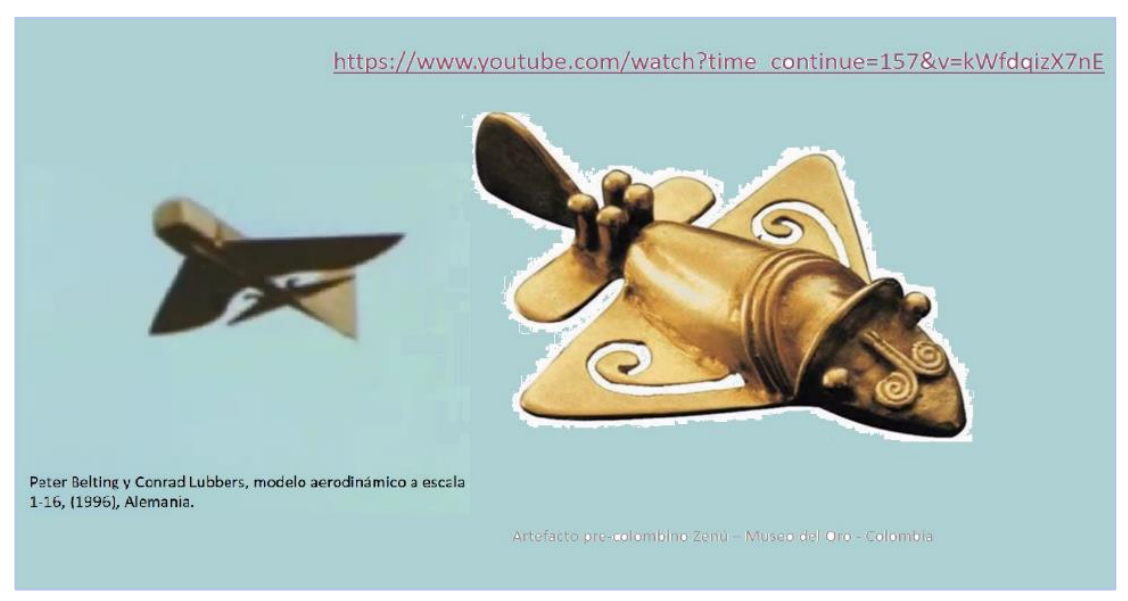

Fuente: Museo del Oro - Bogotá (Colombia) / César + History Channel. (03.12.2009). Aviones Precolombinos, aeromodelismo hace 1000 años. A+E Networks. Recuperado de: <https://www.youtube.com/watch?time continue=157\&v=kWfdqizX7nE>

La imagen representa un modelo a escala $(1-16)$ de uno de los muchos objetos de oro hallados - o más bien robados - en los camellones y asentamientos indígenas, mediante el cual, ingenieros holandeses han logrado demostrar tener propiedades aeronáuticas.

Uno de los enigmas sin resolver, acerca de los camellones pre-hispánicos, es justamente descubrir ¿cómo lograron estas personas, sin aparatos topográficos, sin mapas cartográficos, sin sobrevolar el territorio y, hace más de dos mil años - fecha estimada para la construcción de estos ingenios hidráulicos - trazar y drenar mediante este sistema más de quinientas mil hectáreas de terreno y además hacerlo de manera tal que controlase las frecuentes inundaciones de la hoy llamada "depresión momposina"?

Aunque se logre algún día una explicación "científica" de lo anterior, y habiendo logrado que los camellones indígenas zenúes fuesen considerados como patrimonio inmaterial (y material) de la humanidad, surgiría la inquietud: ¿Para qué sirve la resignificación, en este caso, de objetos patrimoniales que han entrado en desuso?

La re-significación de asentamientos humanos alrededor de ecosistemas estratégicos como patrimonio cultural implica la designación de estos sitios como Sistemas Ingeniosos 
del Patrimonio Alimentario Mundial, lo cual puede ayudar a conservar la biodiversidad y salvaguardar variedades de cultivos y especies animales en peligro de extinción. En un sentido más amplio al significado que la FAO le otorga a los SIPAM ${ }^{7}$, lo agrícola debería incluir el cultivo de las coberturas de agua además de las coberturas del suelo y de esta manera, un ecosistema estratégico puede ser comprendido como Sistema Importante del Patrimonio Alimentario Mundial.

La siguiente figura, ilustra como lograron estos "indígenas" prehistóricos un equilibrio entre el agua y la tierra sin generar bordes, esto es: sin límites aparentes en el territorio, sin segregación social ni fragmentación de asentamientos humanos. Se trata de la cultura "anfibia" que Gustavo Wilches Chaux (2016), describiera en sus intentos por introducir el texto introductorio de la constitución política colombiana en 1991: Colombia es un país: pluriétnico, multicultural y anfibio.

Figura 3. Sistemas ingeniosos del patrimonio alimentario mundial

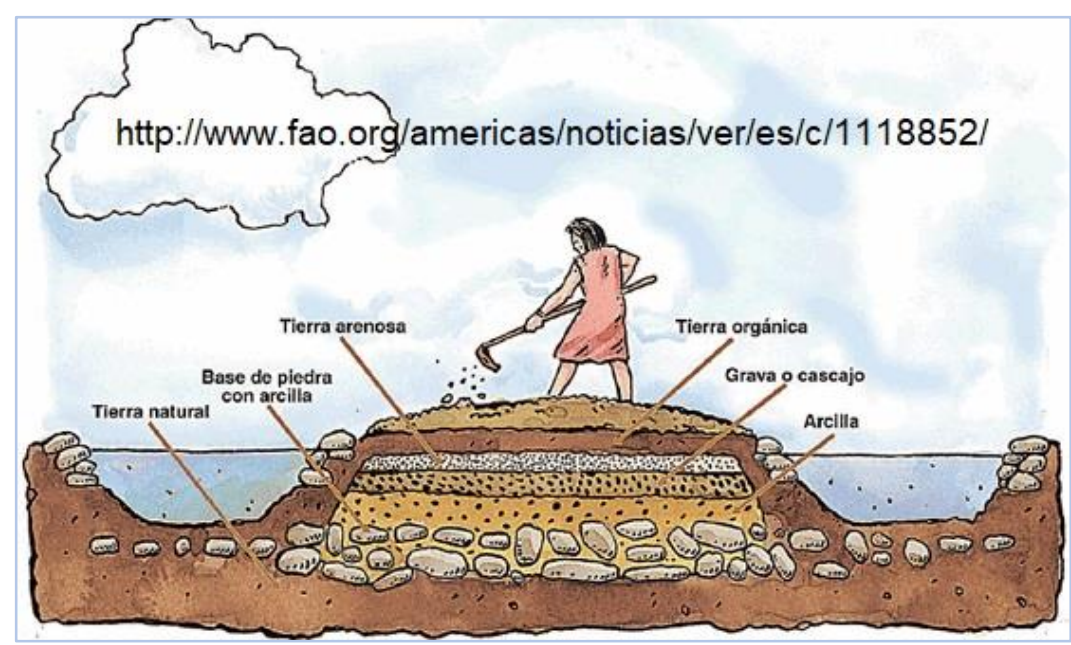

Fuente: Imagen tomada del Blog de Anónimo (27.07.2017). Agricultura, minería y manufactura de los Incas. Lima (Perú): Blogspot. Recuperado de: <http://econinca.blogspot.com/2017/07/agricultura-incaica.html>

$7 \quad$ El término "importante", originalmente era "ingenioso". Interesante resignificar la sigla contrastando el sitio oficial disponible en: <http://www.fao.org/giahs/es/> con el texto de PARVIZ, Koohafkan \& ALTIERI, Miguel. (2011). "Sistemas Ingeniosos del Patrimonio Agrícola Mundial: Un Legado para el Futuro". Roma: FAO/ONU. Recuperado de: <http://www.fao.org/3/i2232s/i2232s.pdf> 
La problemática que aborda la declaratoria de los SIPAM, además de requerir la resignificación del propio término, proviene de los efectos combinados derivados del cambio climático y la pérdida de la biodiversidad. Las migraciones humanas debido a factores económicos y el consecuente abandono de los campos y de las prácticas ancestrales, imposibilitan el aprovechamiento de los recursos manteniendo el cuidado de la naturaleza. En lugares extremadamente bellos del mundo, como en Colombia, se conservan aún estos sistemas alimentarios tradicionales singulares que constituyen la base de innovaciones presentes y futuras y se hace urgente y necesario su conservación y protección.

La estructura de camellones indígenas que genera formas únicas e ingeniosas mediante las cuales se ha alcanzado la sostenibilidad en la actividad humana más básica, convirtiendo los recursos naturales en sistemas alimentarios viables, responde a vibraciones de la materia del agua que almacenan y transmiten memoria.

El resultado de la investigación que el Museo del Oro y el Banco de La República (Mincultura, 2010), han hecho sobre las huellas de los extensos canales de drenaje construidos por los indígenas zenúes hace 2000 años, deja apreciar cómo hicieron estas poblaciones para lograr en un solo sistema una estructura habitable planificada, sistemas productivos sostenibles y además controlar las inundaciones reteniendo el agua necesaria para mantener la productividad durante todos los días del año sin interrupción en los valles de los ríos Sinú, San Jorge y Cauca.

Las siguientes imágenes y sus respectivos enlaces disponibles en la Internet muestran ese tejido del agua y la tierra en un territorio que aún conserva una pequeña parte de dichas estructuras. Podemos imaginar cómo ha sido el proceso de su destrucción cuando el pisoteo del ganado introducido por los conquistadores europeos logró su cometido: desplazar la población original y de paso introducir la ganadería extensiva, la propiedad privada de las tierras y de paso legitimar el saqueo y la extracción masiva del oro. 
Figura 4. Camellones pre-hispánicos Zenúes.

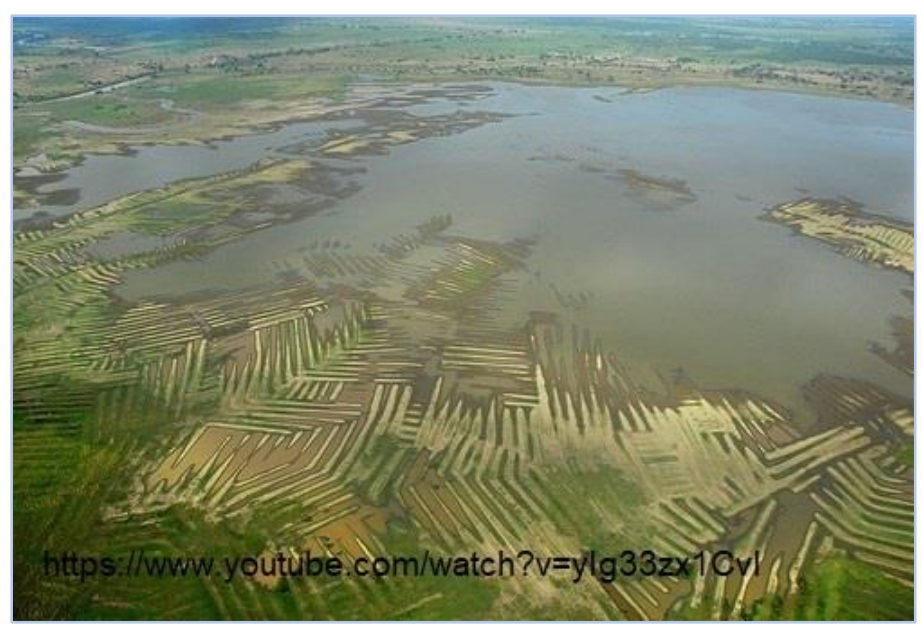

Fuente: Banco de la República. (16.12.2011). El Tejido del Agua. Bogotá: Museo del Oro Colombia. Recuperado de: <https://www.youtube.com/watch?v=ylg33zx1Cvl>

Si se pretende fomentar la conservación "dinámica" y propiciar que "nosotros", descendientes de los originales quienes ayudaron a crear SIPAM, actuemos como custodios de este legado y mantengamos viva la memoria del agua frente a desafíos - como la urbanización y el cambio climático - tendríamos que entender de una manera experiencial la relación entre la memoria del agua y el desdoblamiento del tiempo.

"Serían visiones del futuro o re-memoranzas del futuro pasado. [...] Habría una reestructuración en los cromosomas de la memoria de secretos antiquísimos de nuestros antepasados superiores" Charroux (1976: 201), en referencia al "alma del Universo".

\section{Figura 5. Realidad Desdoblada.}

\section{Realidad "desdoblada".}

Lo pasado (lo que yá pasó) está adelante del Ahora, esperándonos en el Futuro. Hurtado, 1994.

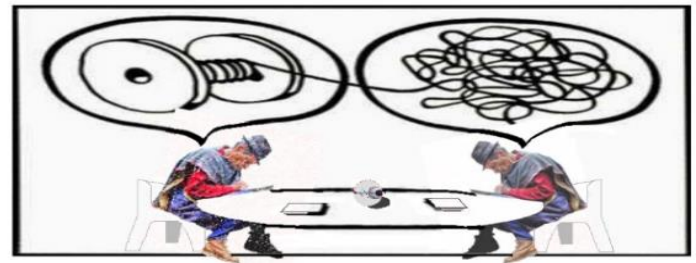

Lo

Pasado

El Ahora

Futuro

Fuente: Imagen alterada del original. Didier Humberto Chirimuskay. (21-12-2015). Otro Espacio para La Memoria. Territorio Wampia - Misak: ONIC - Noticias. Recuperado de: <https://www.onic.org.co/noticias/916-hoy-taita-avelino-dagua-hurtado-partio-al-kansro-meiyeinuk-otro-espacio-para-la-memoria> 
Nuestra lógica racional occidental dominante impide que recordemos el futuro. Siendo así, se hace necesario plantearse la posibilidad radical del desdoblamiento del tiempo ( $y$, por tanto, del espacio) como algo físicamente realizable a diversas escalas fractales de la realidad y relacionar todo esto con la memoria del agua.

Como esto último es una labor tan difícil como ambiciosa, la cual implica enlazar diversos aportes teóricos de vanguardia con otros, en esencia "olvidados" a fuerza de imposición del paradigma occidental de pensamiento dominante, se propone hacerlo de manera sintética, apelando a imágenes y se advierte al lector que la totalidad de ideas hasta aquí puestas en el texto, serán plenamente desarrollada en capítulos de libros posteriores.

\section{Premisa 3: El desdoblamiento del tiempo como fenómeno de "intrincación", implica un espacio que solo existe en el "ahora".}

El procedimiento para responder lo anterior además de semiótico y discursivo es deconstructivo y requiere de asumir la otredad en términos de mí-mismo. Una realidad desdoblada, re-significando el pasado (como artículo), por lo pasado (como pronombre). Lo que ya pasó, lo pasado, está adelante del Ahora, esperándonos en el Futuro. El taita Avelino Dagua (1994), lo explicaba de la siguiente manera:

"Lo que pasó está delante de nosotros, (no detrás). Mi padre, mi abuelo y sus ancestros están adelante esperándonos. Ellos ya pasaron por esta vida e hicieron lo que yo apenas voy a hacer más adelante." Solamente entenderíamos estas sabias palabras si existiésemos (conscientemente), en el Kansro Mei Yeinuk, otro espacio para la memoria. ${ }^{8} \mathrm{O}$ como lo diría Eckhart Tolle, en el ahora?.

“Nunca nada ocurrió en el pasado, ocurrió en el Ahora. Nunca ocurrirá nada en el futuro; ocurrirá en el Ahora." (Tolle, 1997). Si no es posible estar, tampoco lo es ser.

$8 \quad$ Noticias ONIC. Diciembre de 2015. Disponible en: <https://www.onic.org.co/noticias/916-hoy-taitaavelino-dagua-hurtado-partio-al-kansro-mei-yeinuk-otro-espacio-para-la-memoria>

$9 \quad$ TOLLE, Eckhart. (1997). "El Poder del Ahora: una guía para la iluminación espiritual". Barcelona: Editorial Grigalbo. Audiolibro recuperado de: <https://www.youtube.com/watch?v=oMSuXcdwQEA> 
Solamente existe la posibilidad de ir. Ir no requiere hacer y menos tener. No se requiere hacer nada porque el movimiento heliocéntrico en espiral de los cuerpos celestes es fractal, su sentido hacia todas las direcciones y su velocidad infinita. Por tanto, se percibe como la quietud total del ahora.

Si la realidad es como el modelo espiral y fractal, nunca regresamos a ningún lugar, siempre viajamos a velocidades incrementales infinitas por todo el universo. Lo podemos apreciar mejor en la siguiente figura:

Figura 6. Sistema en espiral.

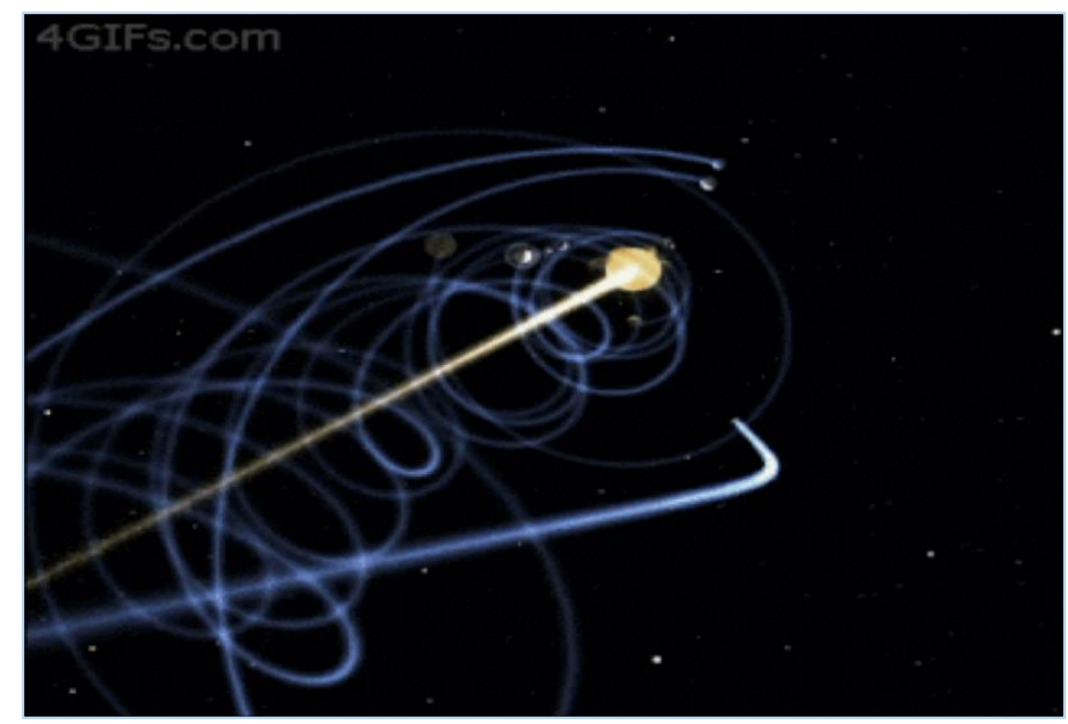

Fuente: Keshava Bhat Pallathadka. (24.12.2012). El modelo de hélice - Nuestro sistema solar es un vórtice. The Netherlands: DjSadhu. Recuperado de:

<https://www.youtube.com/watch?v=0jHsq36 NTU\&feature=youtu.be $>$

Existe una forma geométrica que representa dicha realidad, pero abstraída del movimiento que - supuestamente - existe en virtud del movimiento heliocéntrico en espiral. Se trata del toroide (torus), una estructura de totalidad empaquetada en una forma revolucionada: una curva plana cerrada que gira alrededor de una recta exterior coplanaria con la que no se interseca. 
14. Visión Antataura, Vol.4, No.1, Junio - Noviembre 2020

Figura 7. Torus

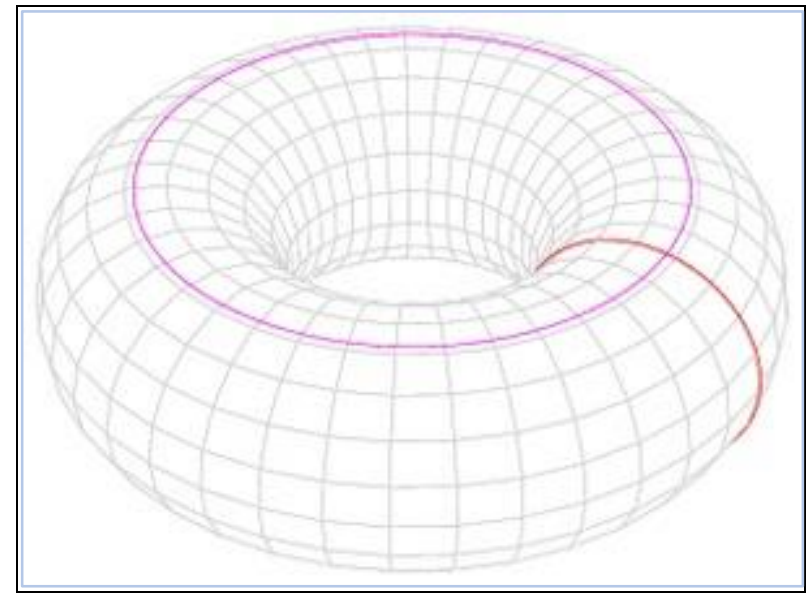

Fuente: Wikipedia. (17.02.2020). Toro (geometría). Recuperado de:

<https://es.wikipedia.org/wiki/Toro (geometr\%C3\%ADa)\#Bibliograf\%C3\%ADa> Se recomienda ver también la siguiente animación:

<https://es.wikipedia.org/wiki/Toro (geometr\%C3\%ADa)\#/media/Archivo:Villarc eau circles.gif>

Donde $R$ es la distancia del eje de revolución al centro de una sección circular del toro y $r$ es el radio de dicha sección.

$$
\begin{aligned}
& A=4 \pi^{2} R r \\
& V=2 \pi^{2} R r^{2}
\end{aligned}
$$

Tanto $A$ (superficie) como $V$ (volumen), son abstracciones que funcionan correctamente en el mundo de la física clásica. Esto es válido a nivel macroscópico, el mundo en el cual, nuestra consciencia como seres humanos puede alcanzar a percibir la velocidad y la posición de una partícula, creando en la mente la ilusión del tiempo lineal que viene del pasado y va hacia el futuro.

Nosotros "somos" un nudo complejo de todo el universo. El "tú" y el "yo", "estamos" intrincados con otros "mí mismo" en ciclos de desdoblamiento del tiempo y del espacio configurados como "paquetes de plenitud". 
Figura 8. Paquetes de Plenitud.

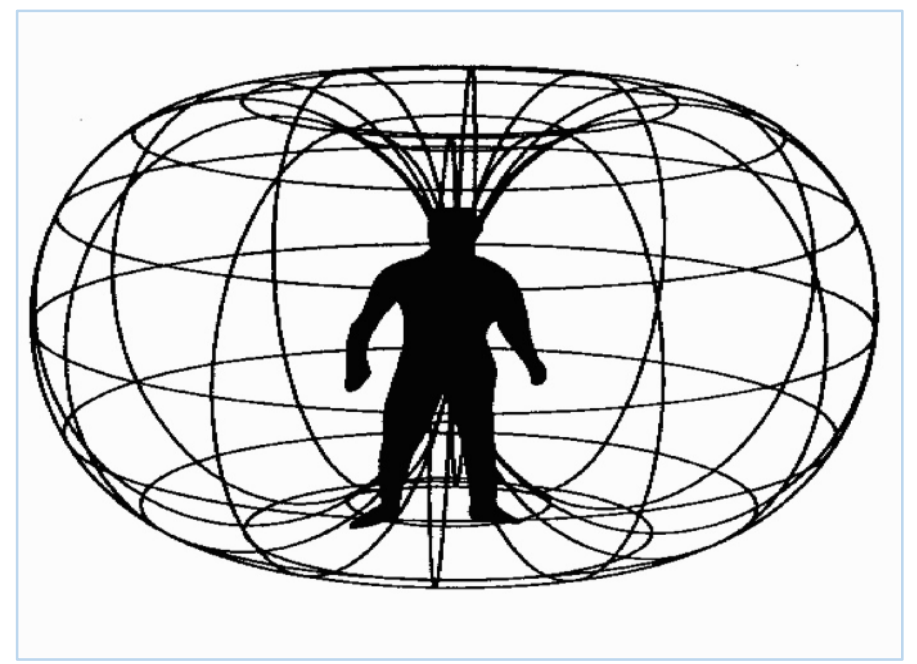

Fuente: Olen Bar der Dunya. (27.03.2019) El Hombre Magnético. Theran (Iran):

Tabnak Javan. Recuperado de: <https://tabnakjavan.com/fa/news/>

El fenómeno de la intrincación está caracterizado por un movimiento en espiral, cuya velocidad se incrementa de manera fractal en cada fase del desdoblamiento de nuestra consciencia temporo-espacial, y es posible calcularla mediante una ecuación que expresa una ley universal. Entender dicho fenómeno es crucial para acceder a un nuevo estado mental en el cual, la física cuántica "genera" la física clásica. Esto significa que la superposición cuántica, aquella que permite entender que un evento observado tenga simultaneidad de alternativas "antes" de ser observado, adquiere coherencia mediante la intrincación.

Figura 9. Desdoblamiento del tiempo (y del espacio).

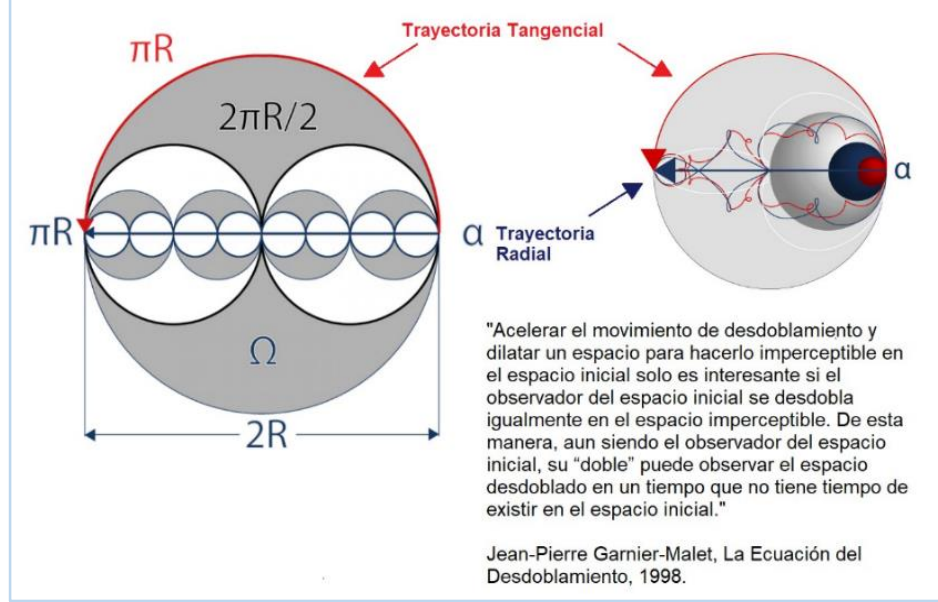

Fuente: Jean Pierre Garnier-Malet. (30.08.2017). La Teoría del Desdoblamiento del Espacio y del Tiempo. Recuperado de: <http://www.garnier-malet.com/es/teoria-deldesdoblamiento/> 
Según el físico Jean Pierre Garnier Malet (2012), todo comienza en un círculo $\Omega$ que gira sobre sí-mismo con una velocidad de rotación constante $\phi$ y cuyo radio es el diámetro de un círculo $\Omega / 2$ dos veces más pequeño que gira sobre sí-mismo dos veces más rápido. Dicho movimiento se acelera el doble del doble en otro círculo $\Omega / 4$ dos veces más pequeño que $\Omega / 2$, y el doble del doble del doble en otro $\Omega / 8$ aún más pequeño y así sucesivamente, (hasta lo infinitamente pequeño). Lo mismo ocurre a la inversa hacia lo infinitamente grande, siendo el circulo $\Omega$, el nivel de consciencia ontológico del ser humano en el mundo de la física "clásica" que hace posible la simultaneidad de estados coherentemente intrincados hacia la realidad cuántica-cósmica.

La coherencia cuántica hace posible que un sistema participe de todos los estados cuánticos posibles sin restricciones de tiempo y espacio siendo, por tanto, un estado general de indefinición ontológica: La alternatividad de lo que todavía no es y al mismo tiempo todo lo es.

¿Cuál sería ese "sujeto consciente", aquel quien haría observable el mundo que, por sí mismo permanecería (de manera imperceptible), en un estado de indefinición cuántica? Nuestra consciencia humana aparentemente perdida en medio de lo infinitamente grande del universo y lo infinitamente pequeño de las partículas. Nosotros habitantes de una tierra que nos parece inmensa, un pequeñísimo planeta de un pequeñísimo sistema solar que nos parece infinito.

Por un cambio de escala de espacio y de tiempo, la ley del desdoblamiento @ reúne lo infinitamente grande de un observador inicial y lo infinitamente pequeño del observador desdoblado. Todo bien hasta ahí, pero y ¿por qué no somos capaces de observar esa realidad desdoblada en el mundo "clásico" del observador (salvo en estados alterados de consciencia)?

Los observadores "conscientes" somos paquetes de plenitud que mediante un acto creativo (del verbo creer más que crear), optamos por una alternativa - de las múltiples posibles - que tiene la alternatividad y, "obligamos" a los sistemas cuánticos a manifestarse en un estado de realidad materialmente observable. 
Si observamos el movimiento del desdoblamiento del tiempo y del espacio y su consecuente apertura entre realidades observables a distintas escalas fractales (borde), veremos, que se trata nuevamente de un toroide, pero fractal: somos paquetes de plenitud a diversas escalas de tiempo-espacio conscientes vagando por el universo a velocidades infinitas, tanto en lo infinitamente grande (cósmico), como en lo infinitamente pequeño (cuántico).

Figura 10. Toroide Fractal

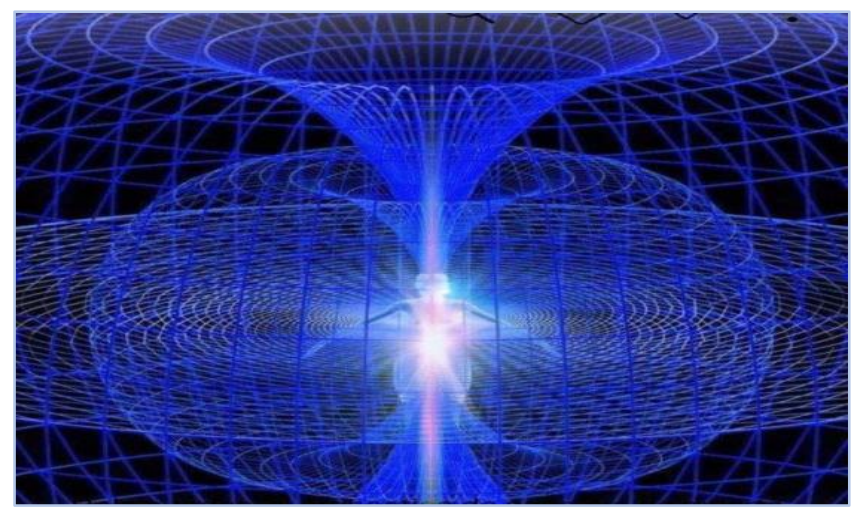

Fuente: Sandra López. (04.12.2019). Canalización de Elsa Farrus, (Kuan Yin. Desde Alcyon). Recuperado de: <http://lamagiadelsilencio.com/canalizacion-de-elsa$\underline{\text { farrus/> }}$

Dicho toroide fractal, surge de un movimiento de desdoblamiento. Un círculo ' $\Omega$ gira sobre sí mismo y su radio gira con una velocidad constante $\phi$. Dicho radio es también el diámetro de un círculo' $\Omega / 2$ dos veces más pequeño que gira por tanto dos veces más rápido sobre sí mismo y en el espacio, alrededor de su diámetro con la misma velocidad $\phi$.

El físico Nassim Haramein (2005), quien, en 1997 formó parte del Sequoia Symposium $^{10}$, relaciona el movimiento toroidal de la consciencia con el efecto Coriolis, descrito por Gaspard-Gustave Coriolis en 1836. Dicha tertulia, organizada por Louis Kauffman, Foster Gamble, Lynnclaire Dennis, Jim Fournier y Ralph Bunje sirvió como marco

10 El Simposio de Sequoia: Explorando Patrones Fundamentales de la Unidad Dinámica, fue un diálogo transdisciplinario, cuyos resultados pueden ser apreciados en el documental organizado por James FosterGamble. (05.04.2012). THRIVE: What On Earth Will It Take? New York: THRIVE Movement. Recuperado de: $<$ https://www.youtube.com/watch?v=IEV5AFFcZ-s\&feature=youtu.be $>$ 
teórico (Dennis y otros, 2009), para analizar el desarrollo, la operación y los retos para sobrevivir de todas las cosas, agrupaciones vivas, no vivas u organizadas.

El efecto Coriolis, describe el movimiento desdoblado de una partícula en movimiento que se encuentra al interior de un cuerpo también en movimiento. La "ilusión" del efecto es producida por la fuerza inercial de la partícula respecto a la masa del cuerpo que la "arrastra". El resultado es un doble movimiento simultáneo en espiral que hace que, por ejemplo, el agua que se desagua al interior de un recipiente situado en el hemisferio norte de nuestro planeta gire en un sentido contrario al agua que se desagua en un recipiente situado en el hemisferio sur del mismo.

Este movimiento, trasladado a escalas fractales, se puede observar tanto en lo infinitamente pequeño del nivel cuántico como en lo infinitamente grande del nivel cósmico de la materia. Esto último debido también al entrelazamiento o fenómeno de intrincación descrito en páginas anteriores, nos permite comprobar, de manera teórica, que el universo conocido se mueve - todo - en espirales que se desenvuelven ( $y$ por tanto también se envuelven), alrededor de otras espirales cada vez mayores (y menores también), lo cual solo puede ser apreciado a escalas fractales.

Figura 11. Espiral toroidal fractal sometida al efecto coreolis.
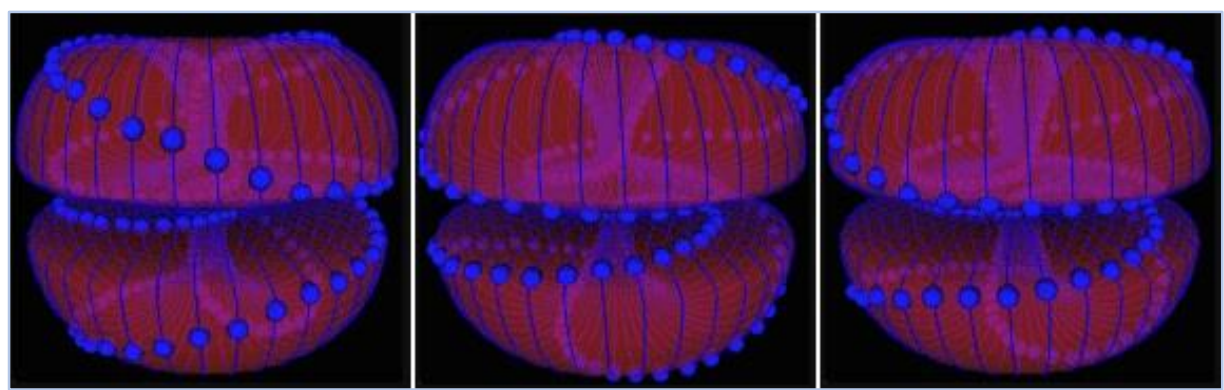

Fuente: Nassim Haramein. (27.08.2010). Verdadera forma del universo. El Blog Alternativo. Recuperado de: https://i0.wp.com/www.elblogalternativo.com/wp-content/uploads/2010/08/nassimtoro.gif?resize $=185 \% 2 \mathrm{C} 175>$

\section{Conclusiones y recomendaciones.}

- La capacidad del agua de retener pensamientos y emociones es la que permite plantear la posibilidad de re-significar formas y comportamientos de los objetos y sujetos en el hábitat. 
- La re-significación de ecosistemas estratégicos en términos de paisaje cultural y la utilidad de éstos últimos como sistemas importantes del patrimonio alimentario mundial, es una propuesta de sustentabilidad del hábitat basada, entre muchas otras ideas, en la teoría de la memoria del agua. Se trata de una teoría de vanguardia que se basa en las matemáticas fractales para entender el universo holográfico y en la geometría sagrada para entender la forma y el movimiento del universo conocido.

- Recuperar el comportamiento original, la maternidad en la mujer y la paternidad en el hombre sin necesidad de poseer, dominar y apropiarse de lo ajeno, sin experimentar el dolor, el pecado y la culpa - todo alternativamente a la idea del desarrollo capitalista y consumista -, es posible mediante un diálogo interior, una negación de la negación de la utopía, reemplazando la nostalgia de recuperar el pasado por la creación de lo pasadofuturo en el ahora, a través de las aperturas temporo-espaciales de la consciencia.

- Un esfuerzo teórico adicional, al expresado por el autor - públicamente en la pasada conferencia del CRUA en Chitré (Panamá) -, asume que los agujeros negros son simultáneamente los límites y los centros del multiverso y que existen a diversas escalas fractales de consciencia: en la estructura de las galaxias y planetas y en los átomos y a nivel de sus partículas cuánticas, siendo realmente nosotros mismos.

- Es la consciencia extendida la que nos hace responsable por ser conscientes de existir y, por extensión, responsables de toda la existencia.

- La memoria del agua nos remite a un universo consciente de un ser humano consciente de su entorno cósmico, cuyas creencias crean coherencia cuántica, que nos ayudan a evolucionar sabiendo que estamos evolucionando, a entender que hay un plan evolutivo y que somos responsables de nuestros actos.

- La mayor utilidad de la memoria del agua como teoría entendida a la luz de la ley del desdoblamiento del tiempo (Garnier-Malet, 2012), consiste en transformar nuestra mirada acerca de lo que miramos y, de esta manera, escapar del apocalipsis futuro que ha sido configurado como tendencia de pasado a presente.

- Para la ortodoxia científica las teorías del físico Nassim Haramein acerca del efecto coriolis en el movimiento fractal del universo y del movimiento de todas sus partículas 
"empaquetadas" a diversas escalas de consciencia "podrían incluirse en el mismo saco que la homeopatía, el biomagnetismo, la numerología, la teoría de los campos mórficos, del diseño inteligente $y$, otros temas que cuestionan, con fundamento o sin él, las verdades oficiales"11.

- Algo no probado por la ciencia oficial de hoy podría ser la base del paradigma del mañana $y$, en consecuencia, dedicarnos a combatir por combatir las ideas que no nos parecen correctas y, hacerlo de una manera sistemática y premeditada (esto es, incluyendo contenidos en la Internet para luego desprestigiarlos y ponerlos en duda), es una labor, no por necesaria, menos desagradecida.

- La calidad de la vibración de las emociones que los lectores de las anteriores líneas han sentido (si han logrado llegar a leer hasta este punto), así como la visualización de las imágenes y la búsqueda juiciosa de las referencias citadas será la prueba (aún no demostrada, pero no por ello menos científica), de que habitamos un universo desdoblado, que no podemos percibir de manera inconsciente ya que se requiere de una onda vibratoria que sintonice nuestros cerebros con dicha realidad.

- La manera como cualquier humano logre lo anterior es asunto de cada quien. Sin embargo, el autor, recomienda al lector usar la mente racional y, no por ello demerita ningún otro método noético ${ }^{12}$ para llegar al conocimiento, siempre y cuando no atente contra cualquier forma de vida o aliente al deterioro innecesario de la vida misma.

- La última figura en este escrito se refiere al mundo Zenú, conocido como Panzenú. Se puede observar en esta vasija ceremonial el trazado de los camellones hidráulicos y la manera como la técnica de su entrelazamiento y disposición geográfica ha sido

11 Acerca de comentarios a favor y en contra de la pseudociencia y su importancia en la Internet, se recomienda comenzar por leer los comentarios del Bibliotecario de la Wikipedia, Enrique Sánchez Ludeña. (28.12.2016). Nassim Haramein y la Wikipedia. Madrid: Wikipedia. Recuperado de: <https://www.otraspoliticas.com/educacion/nassim-haramein-y-la-wikipedia/>

12 La noética estudia el conocimiento inteligible, pensable por el intelecto y hace parte, tanto de la doctrina aristotélica occidental como del pensamiento intuitivo, interior, oriental. Acerca de publicaciones noéticas contemporáneas se recomienda visitar el sitio: Richard L. Amoroso. (24.01.1998-2008). Publicaciones Noéticas. Oakland, CA (USA) : The Noetic Press. Recuperado de: <http://www.mindspring.com/ noeticj/> 
cuidadosamente diseñada y su conocimiento ancestral ha sido inteligentemente encriptado en las figuras del entrelazamiento de caña flecha que configuran las vueltas del sombrero típico de la región, a la vista de todos, pero secretamente guardado para que algún día dicho conocimiento sea revelado a la humanidad.

Figura 12. Mundo Zenú.

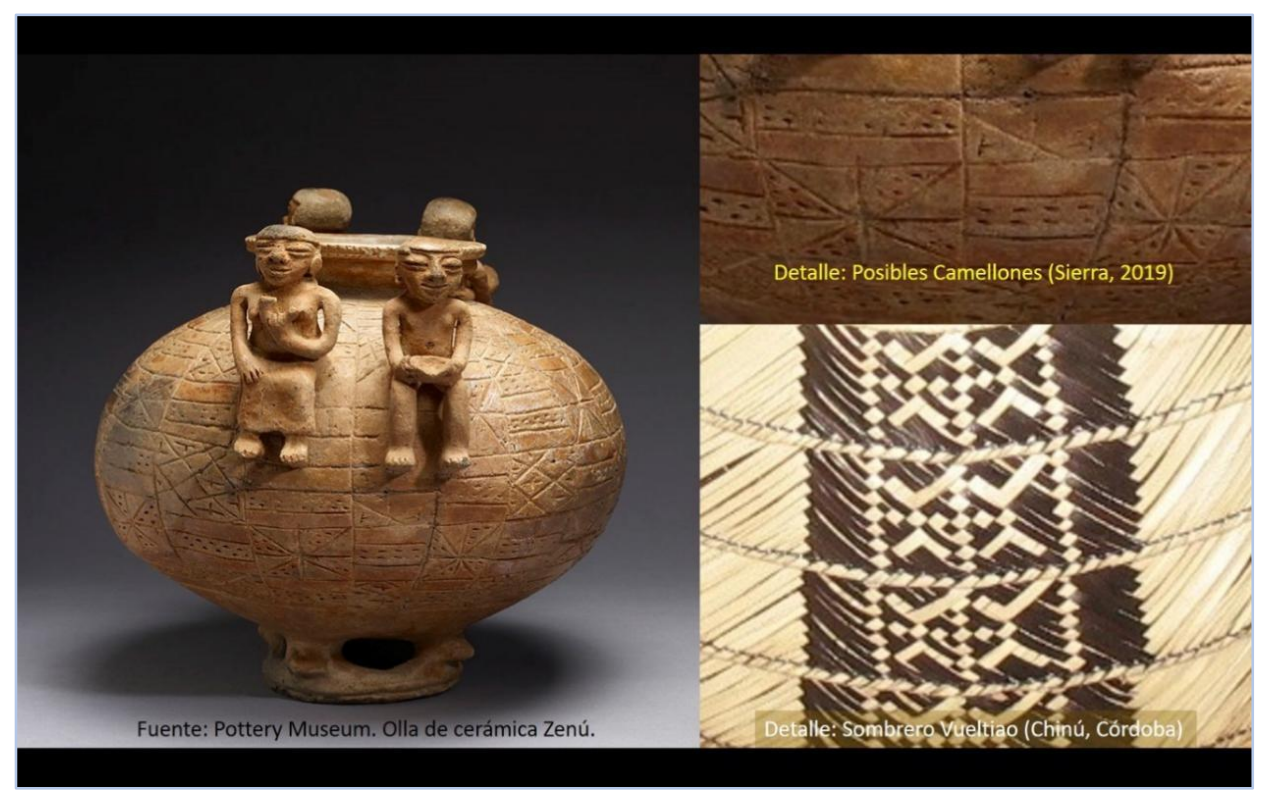

Fuente: Pottery Museum. A la derecha, detalle del sombrero Vueltiao, patrimonio colombiano.

\section{Referencias bibliográficas}

Amoroso, R. L. (24.01.1998-2008). Publicaciones Noéticas. Oakland, CA (USA): The Noetic Press. Recuperado de: <http://www.mindspring.com/ noeticj/>

Anónimo (27.07.2017). Agricultura, minería y manufactura de los Incas. Lima (Perú):

Blogspot. Recuperado de: <http://econ-inca.blogspot.com/2017/07/agriculturaincaica.html>

Anónimo. (02.10.2019). El Poder del Ahora (Audiolibro completo) Eckart Tolle. Youtube: Blog Anónimo. Recuperado de: <https://www.youtube.com/watch?v=oMSuXcdwQEA $>$

Asta. (17.03.2020). Orgasmic Birth. The Best-Kept Secret - The Film. New York: Orgasmic Birthing. Recuperado de: <https://www.orgasmicbirth.com/products/filmssoundtrack/> 
Banco de la República. (16.12.2011). El Tejido del Agua. Bogotá: Museo del Oro Colombia. Recuperado de: <https://www.youtube.com/watch?v=ylg33zx1Cvl>

Bar der Dunya, O. (27.03.2019) El Hombre Magnético. Theran (Iran): Tabnak Javan. Recuperado de: <https://tabnakjavan.com/fa/news/>

Bhat Pallathadka, K. (24.12.2012). El modelo de hélice - Nuestro sistema solar es un vórtice. The Netherlands: DjSadhu. Recuperado de: <https://www.youtube.com/watch?v=0jHsq36 NTU\&feature=youtu.be>

Charroux, R. (1976). "El libro de los mundos olvidados". Barcelona: Plaza \& Janes Editores. Chirimuskay, D. H. (21-12-2015). Otro Espacio para La Memoria. Territorio Wampia - Misak: ONIC - Noticias. Recuperado de: <https://www.onic.org.co/noticias/916-hoy-taitaavelino-dagua-hurtado-partio-al-kansro-mei-yeinuk-otro-espacio-para-la-memoria>

De Coriolis, G. G. (1832). “Memoire sur le principe des forces vives dans les mouvements relatifs des machines". Dans: journal de l'École Polytechnique, v. XIII, cahier XXI (1832), p. 268-302. Recuperado de: <http://www.bibnum.education.fr/physique/mecanique/sur-les-equations-dumouvement-relatif-des-systemes-de-corps>

Dennis, L., Gray, R.W., Kauffman, L.H., Brender, J. (2009). “A Framework Linking Non-living and Living Systems: Classification of Persistence Survival and Evolution Transitions". Foundations of Science. Recuperado de: $<$ https://www.researchgate.net/publication/225492645 A Framework Linking No nLiving and Living Systems Classification of Persistence Survival and Evolution T ransitions>

Emoto, M. (2012). Mensajes del agua - La belleza oculta del agua. Barcelona: Editorial La Liebre de Marzo. Recuperado de: $<\underline{\text { https://gestionsostenibledelagua.files.wordpress.com/2012/07/mensajes de agua }}$ .pdf> 
23. Visión Antataura, Vol.4, No.1, Junio - Noviembre 2020

Foster-Gamble, J. (05.04.2012). THRIVE: What On Earth Will It Take? New York: THRIVE Movement. Recuperado de: <https://www.youtube.com/watch?v=|EV5AFFcZs\&feature=youtu.be>

Garnier, J. P. \& Malet, L. (2012). “Cambia tu futuro por las aperturas temporales". Paris: Corine Leblanc Ed. Recuperado de: <http://www.garnier-malet.com/es/teoria-deldesdoblamiento/>

Garnier-Malet, J. P. (30.08.2017). La Teoría del Desdoblamiento del Espacio y del Tiempo. Recuperado de: <http://www.garnier-malet.com/es/teoria-del-desdoblamiento/>

Haramein, N. and Rauscher, Elizabeth. (2005). "The origin of spin: A consideration of torque and coriolis forces in Einstein's field equations and Grand Unification Theory", in R. L. Amoroso, B. Lehnert \& J-P Vigier (eds.) Beyond The Standard Model: Searching For Unity In Physics. The Noetic Journal. 2005, pp. 153-168. Recuperado de: < http://omega432.com/wordpress/wpcontent/uploads/2014/05/torque paper.pdf>

Haramein, N. (27.08.2010). Verdadera forma del universo. El Blog Alternativo. Recuperado de: <https://i0.wp.com/www.elblogalternativo.com/wpcontent/uploads/2010/08/nassim-toro.gif?resize=185\%2C175>

History Channel. (03.12.2009). Aviones Precolombinos, aeromodelismo hace 1000 años. $A+E$ Networks. César Castillo. Recuperado de: <https://www.youtube.com/watch?time continue=157\&v=kWfdqizX7nE >

Igual, B. (26.04.2017). ¿Qué es la Oxitocina?: Blog CuerpoMente. Recuperado de: <https://www.cuerpomente.com/salud-natural/terapias-naturales/que-eshormona-oxitocina-para-que-sirve 264>

López, S. (04.12.2019). Canalización de Elsa Farrus, (Kuan Yin. Desde Alcyon). Recuperado de: <http://lamagiadelsilencio.com/canalizacion-de-elsa-farrus/>

Lovelock, J. (1985). "Gaia, una nueva visión de la vida sobre la Tierra". Barcelona: Ediciones Orbis. Recuperado de: <http://mateandoconlaciencia.zonalibre.org/gaia.pdf>

Ministerio de Cultura (2010) "Zenú, la gente de la palabra". Bogotá: Editorial del Banco de la República. Recuperado de: 
24. Visión Antataura, Vol.4, No.1, Junio - Noviembre 2020

$<\underline{\text { https://www.mincultura.gov.co/areas/poblaciones/noticias/Documents/Caracteriz }}$ aci\%C3\%B3n\%20del\%20pueblo\%20Zen\%C3\%BA.pdf>

Northrup, C. (2010). "Cuerpo de mujer, sabiduría de mujer". Bogotá: Ediciones Urano.

(Sinopsis) recuperada de: <http://edicionesuranocolombia.com/es-

ES/catalogo/catalogo/cuerpo de mujer sabiduria de mujer001000176 ? id $=001000176>$

Olza, I. (23.10.2018). Charla Casilda Rodrigañez. Barcelona: Instituto Europeo de Salud Mental Perinatal. Recuperado de: $<$ https://www.youtube.com/watch?time continue=14\&v=T9kBRA0RQ38>

Parviz, K. \& Altieri, M. (2011). "Sistemas Ingeniosos del Patrimonio Agrícola Mundial: Un Legado para el Futuro". Roma: FAO/ONU. Recuperado de: $<$ http://www.fao.org/3/i2232s/i2232s.pdf >

Rodrigañez, C. (2001). "El asalto al Hades - La rebelión de Edipo". Barcelona: Traficantes de Sueños. Recuperado de: <https://pt.scribd.com/document/431225062/Edipo-II-Elasalto-a-Hades-Casilda-Rodriganez>

Sánchez, E. (28.12.2016). Nassim Haramein y la Wikipedia. Madrid: Wikipedia. Recuperado de: < $<$ https://www.otraspoliticas.com/educacion/nassim-haramein-y-la-wikipedia/

Tolle, E. (1997). "El Poder del Ahora: una guía para la iluminación espiritual". Barcelona: Editorial Grigalbo. Audiolibro recuperado de: <https://www.youtube.com/watch?v=oMSuXcdwQEA>

Verónica. (21.07.2011). La consciencia afecta a la materia. Masuro Emoto. Chile: Blog de crecimiento personal. Recuperado de: $<\underline{\text { http://mujerayuda.blogspot.com/2011/07/la-consciencia-afecta-la-materia- }}$ masaru.html>

Wikipedia. (17.02.2020). Toro (geometría). Recuperado de: $<$ https://es.wikipedia.org/wiki/Toro (geometr\%C3\%ADa)\#Bibliograf\%C3\%ADa>. Se recomienda ver también la siguiente animación: $<\underline{\text { https://es.wikipedia.org/wiki/Toro (geometr\%C3\%ADa)\#/media/Archivo:Villarceau }}$ circles.gif> 